\title{
Albuterol Delivery Efficiency in a Pediatric Model of Noninvasive Ventilation With Double-Limb Circuit
}

\author{
Jeanne Velasco MD and Ariel Berlinski MD
}

\begin{abstract}
BACKGROUND: Noninvasive ventilation (NIV) is used to treat respiratory failure in patients with concomitant need for aerosol delivery. Limited pediatric data are available on aerosol delivery efficiency, and none at all regarding aerosol delivery efficiency with a double-limb circuit. We compared the effect of position in the double-limb ventilator circuit, types of nebulizer, and ventilator settings on aerosol delivery efficiency in a pediatric model of NIV. We hypothesized that placing a vibrating mesh nebulizer at the ventilator and using the highest inspiratory pressures would increase aerosol delivery efficiency. METHODS: A breathing simulator was connected in series to a low dead-space filter holder (lung dose) and to an anatomically correct head/airway model of a 5-year-old child. A non-vented mask connected the model to a ventilator operated on noninvasive bi-level mode and assembled with a double-limb, heated-wired adult circuit. Inspiratory pressures of either 15 or $20 \mathrm{~cm} \mathrm{H}_{2} \mathrm{O}$ and an expiratory pressure of $5 \mathrm{~cm} \mathrm{H}_{2} \mathrm{O}$ were used. Two different vibrating mesh nebulizers and 2 different jet nebulizers loaded with albuterol solution were studied. Albuterol was measured with spectrophotometry. Aerosol delivery efficiency was calculated as lung dose expressed as a percentage of the nominal dose. RESULTS: A vibrating mesh nebulizer before the mask or Y-piece provided the highest delivery efficiency and outperformed a vibrating mesh nebulizer integrated into the mask. Vibrating mesh nebulizers delivered more drug than jet nebulizers, regardless of their position in the circuit. Increasing the inspiratory pressure only improved aerosol delivery efficiency with a jet nebulizer placed at the ventilator. CONCLUSIONS: In a pediatric model of NIV, the effect of nebulizer position on aerosol delivery efficiency depends on the type of device and its placement in the ventilator circuit. A vibrating mesh nebulizer placed at the mask or before the Y-piece of the double-limb circuit provided the highest aerosol drug delivery during NIV. Data generated with invasive ventilation models should not be generalized to NIV models. Key words: aerosol delivery; noninvasive ventilation; nebulizer; airway model; lung dose; pediatrics; albuterol. [Respir Care 2018;63(2):141-146. @ 2018 Daedalus Enterprises]
\end{abstract}

\section{Introduction}

Noninvasive ventilation (NIV) is often used in the treatment of pediatric respiratory failure, including patients

\footnotetext{
Dr Velasco is affiliated with University of Arkansas for Medical Sciences, Department of Pediatrics, Pulmonology Section, Little Rock, Arkansas. Dr Berlinski is affiliated with University of Arkansas for Medical Sciences, Department of Pediatrics, Pulmonology Section, and with the Pediatric Aerosol Research Laboratory, Arkansas Children's Research Institute, Little Rock Arkansas.
}

The study was supported by the University of Arkansas for Medical Sciences College of Medicine Children's University Medical Group Fund Grant Program (\#036167). The Pediatric Aerosol Research Laboratory at with asthma who do not respond to conventional therapies. ${ }^{1,2}$ NIV provides relief from airway obstruction by stenting the airways open, reducing the work of breathing

\footnotetext{
Arkansas Children's Research Institute was partially established and receives partial support from the George Endowment for Asthma.

Dr Velasco presented an earlier version of this work at the 2016 American Thoracic Society International Meeting, held May 13-18, 2016, in San Francisco, California.

Dr Berlinski has relationships with AbbVie, Anthera, Aptalis Pharma, Cempra, Janssen Research and Development, Gilead, National Institutes of Health, Novartis, and Therapeutic Development Network, Vertex, and the International Pharmaceutical Aerosol Consortium on Regulation and Science. Dr Velasco has disclosed no conflicts.
} 
and intrinsic PEEP, and increasing alveolar ventilation. ${ }^{1}$ Many of these patients also receive inhaled aerosols.

NIV can be delivered via different ventilators that use either a single-limb or a double-limb ventilator circuit. A single-limb circuit uses an exhalation port to allow exhaled

See the Related Editorial on Page 256

gases from the breathing circuit to exit to the atmosphere..$^{3,4}$ The high gas flow and the common inhalation/exhalation pathway in a single-limb circuit are likely to cause higher turbulent flow in the system. ${ }^{5}$ A double-limb circuit has an inspiratory limb and an expiratory limb. There are data on the effect of aerosol generator choice and position in the circuit during invasive ventilation using a heated-wire double-limb circuit. The configuration of the circuit has the potential to influence aerosol delivery. The vibrating mesh nebulizer was found to be the most efficient device, and placement at the ventilator was the most efficient position. ${ }^{6} \mathrm{We}$ are unaware of any published data on aerosol delivery on noninvasive mode using a double-limb circuit in a pediatric model.

Jet and vibrating mesh nebulizers are commonly used to deliver aerosolized medications to patients receiving NIV. 5,7-13 Jet nebulizers use a gas flow to convert a solution/suspension into an aerosol. ${ }^{14}$ Vibrating mesh nebulizers produce an aerosol by vibrations of a mesh or a plate with multiple apertures. ${ }^{14}$ Several adult studies of NIV using a single-limb circuit have shown that a vibrating mesh nebulizer positioned between the exhalation port and the lung model provides efficient drug delivery. ${ }^{7-13}$ A study using an anatomically correct pediatric model and a single-limb circuit showed that delivery of albuterol was most efficient when the vibrating mesh nebulizer was integrated into the mask. ${ }^{5}$

An in vitro adult study using a single-limb circuit showed that aerosol delivery increased as inspiratory positive airway pressure (IPAP) was increased when the nebulizer was positioned after the exhalation port. ${ }^{7} \mathrm{We}$ are unaware of any published data on the effect of different ventilator settings on albuterol delivery during NIV in a pediatric model.

The most efficient device and the best placement of the device on the ventilator circuit during NIV are not known for pediatric patients. There are some published data on adult models, and one study on a pediatric model. Many of these

Correspondence: Ariel Berlinski MD, University of Arkansas for Medical Sciences, Department of Pediatrics, Pulmonary Medicine, 1 Children's Way, Slot 512-17, Little Rock, AR 72202. E-mail: berlinskiariel@uams.edu.

DOI: $10.4187 /$ respcare. 05833

\section{QUICK LOOK}

\section{Current knowledge}

Delivery efficiency of various aerosol generators placed in different positions on a double-limb circuit during pediatric noninvasive ventilation and the effect of different inspiratory pressure are unknown.

\section{What this paper contributes to our knowledge}

Vibrating mesh nebulizers were more efficient than jet nebulizers during pediatric noninvasive ventilation using a double-limb ventilator circuit with a non-vented mask. Devices were more efficient when placed closer to the model, but this did not improve efficiency when the inspiratory pressure was increased.

studies have limitations such as use of a single-limb ventilator circuit, use of only one type of device, and use of nonanatomically correct models.5,7-13 Data on aerosol delivery efficiency of different devices when inserted at different places on the ventilator circuit is clinically relevant. Our institution uses a double-limb circuit during NIV. Obtaining these data are clinically relevant because intubated pediatric patients are frequently extubated to NIV while their need for inhaled therapy is still present.

In this study, we compared the effect of different positions in the ventilator circuit, different types of nebulizers, and different ventilator settings in aerosol delivery efficiency by using an anatomically correct in vitro model of a spontaneously breathing child receiving NIV with double-limb circuit. We hypothesized that using a vibrating mesh nebulizer, placing the nebulizer at the ventilator, and the using the highest IPAP will increase aerosol delivery efficiency.

\section{Methods}

\section{Interface}

A non-vented oronasal mask (small size AF531, Philips Respironics, Murrysville, Pennsylvania) with interchangeable elbow adapter was used (Fig. 1). ${ }^{5}$

\section{Delivery Devices}

Four units of 4 different nebulizers were tested (Fig. 1). We used 4 different nebulizers including 2 different models of aerosol generators. We used a vibrating mesh nebulizer (Solo, Aerogen, Galway, Ireland) that is placed in the ventilator circuit with a T-piece and a vibrating mesh nebulizer that is integrated into the mask (NIVO, Aerogen, Galway, Ireland). The continuous output jet nebulizers tested were the Hudson Updraft II Opti-Neb (Teleflex Med- 


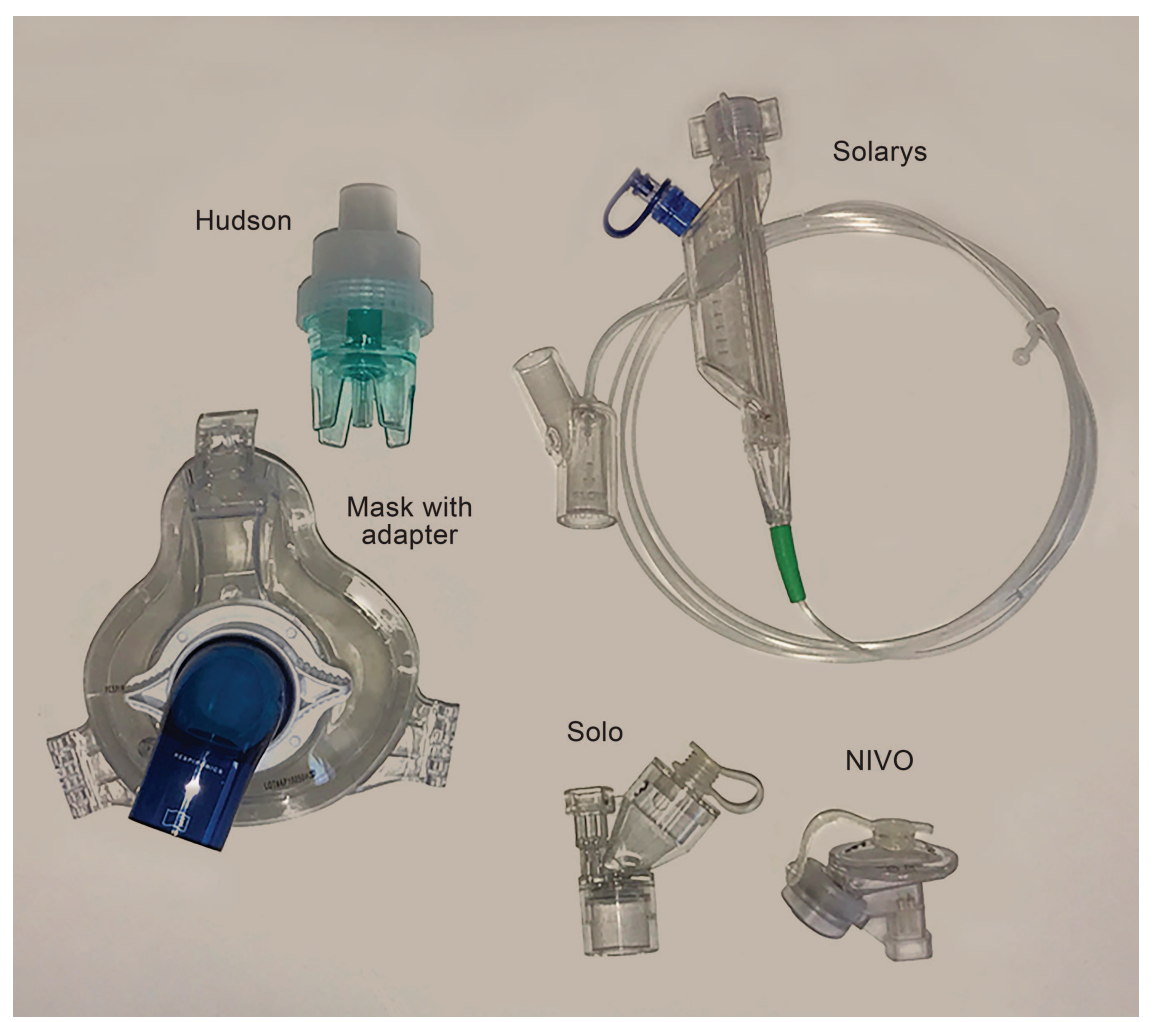

Fig. 1. Interface and delivery devices used in the study.

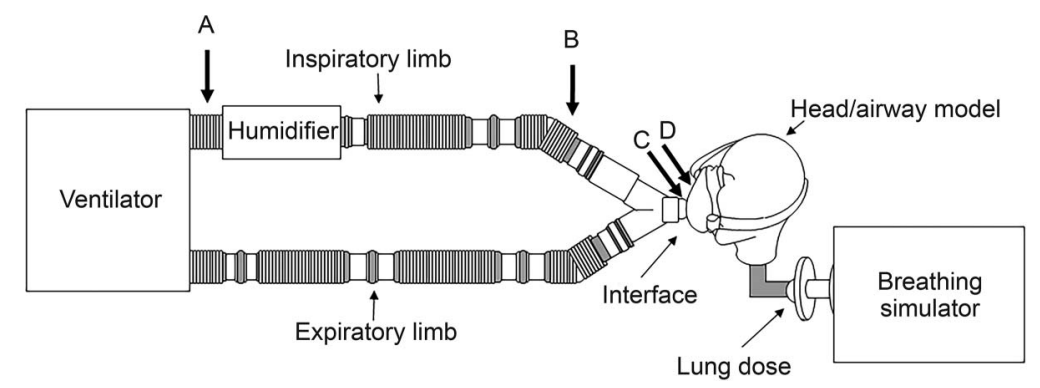

Fig. 2. Position A: At the ventilator on the inspiratory side. Position B: In the inspiratory limb, before the Y-piece. Position C: Between the Y-piece and the elbow that connects to the interface. Position D: Incorporated to the elbow that connects to the interface.

ical, Research Triangle Park, North Carolina) operated at $6 \mathrm{~L} / \mathrm{min}$, and Solarys (Monaghan Medical, Plattsburg, New York) operated at $1 \mathrm{~L} / \mathrm{min}$. Solarys is a low-flow aerosol delivery system that produces continuous nebulization at the distal tip of a multi-lumen catheter that is readily interfaced to the breathing circuit. ${ }^{15}$ The Hudson nebulizer requires higher flows to operate in optimal conditions.

\section{In Vitro Model of a Spontaneously Breathing Child}

The breathing model was composed of a breathing simulator (Dual Phase Control Respirator, Harvard Apparatus, Holliston, Massachusetts) operated with a pediatric breathing pattern (tidal volume $=200 \mathrm{~mL}$, breathing fre- quency $=20$ breaths/min, inspiratory to expiratory time ratio $1: 3$, and inspiratory time $0.75 \mathrm{~s}$ ), connected in series to a low dead-space filter holder (lung dose) and to an anatomically correct head/airway 3-dimensional model of a 5-year-old child (Fig. 2). ${ }^{16}$ The head/airway model was downloaded from www.rddonline.com. The breathing pattern parameters are representative of pediatric age, and allowed comparison to previously published data obtained during invasive mechanical ventilation. ${ }^{6}$

\section{Ventilator Settings}

A Servo-i ventilator (Maquet Critical Care, Solna, Sweden) connected to a humidifier and an adult-sized double- 
limb heated wire circuit (Evaqua, Fisher \& Paykel, Auckland, New Zealand) was used. The Y-piece was connected to the oronasal mask (Fig. 1). The ventilator was operated on the noninvasive bi-level mode with IPAP of $15 \mathrm{~cm}$ $\mathrm{H}_{2} \mathrm{O}$, expiratory positive airway pressure (EPAP) $5 \mathrm{~cm} \mathrm{H}_{2} \mathrm{O}$, and a back-up frequency of 15 breaths/min. To test the effect of 2 different IPAP and EPAP settings on albuterol delivery, we also operated it with IPAP of $20 \mathrm{~cm} \mathrm{H}_{2} \mathrm{O}$ and EPAP of $5 \mathrm{~cm} \mathrm{H}_{2} \mathrm{O}$ with back up frequency of 15 breaths/min.

\section{Study Procedure}

The study was performed at the Pediatric Aerosol Research Laboratory of Arkansas Children's Research Institute in Little Rock, Arkansas. The breathing simulator was connected to the low dead-space filter holder. A new aerosol filter (Pari, Pari Respiratory Equipment, Midlothian, Virginia) was used for each experiment. The breathing pattern was programmed, and the accuracy of the tidal volume was verified with a mass flow meter (TSI 4043, Shoreview, Minnesota) and its associated software. ${ }^{17}$ The head/airway model was connected to the filter, and the breathing parameters were rechecked. The interface was placed on the head model and connected to the Servo-i ventilator through the ventilator circuit. A commercially available gel mask was placed between the face and the mask to allow a good seal. Noninvasive bi-level positive airway pressure mode was set in the Servo-i ventilator. Four units of 4 different brands of nebulizers loaded with albuterol sulfate solution $(2.5 \mathrm{mg} / 3 \mathrm{~mL})$ were tested.

Hudson and Solarys nebulizers were connected to a central air source (50 pounds per square inch) and a regulated flow meter. The Hudson nebulizer was operated for $5 \mathrm{~min}$ as per previous evaluations, and the others were operated for 15 min. ${ }^{6}$ The Hudson and the Solo nebulizers were placed before the Y-piece (Fig. 2B) and at the ventilator (Fig. 2A). The Solo was also placed between the Y-piece and the mask. The NIVO was placed in the elbow that connects the interface to the Y-piece (Fig. 2D), and the Solarys was placed between the Y-piece and the elbow that connects the interface (Fig. 2C). The placement of the different devices was made to allow comparison to previous studies. ${ }^{5,6}$ The study was first completed with IPAP/EPAP of $15 / 5 \mathrm{~cm} \mathrm{H}_{2} \mathrm{O}$, and then with IPAP/EPAP of $20 / 5 \mathrm{~cm} \mathrm{H}_{2} \mathrm{O}$. Upon completion of the study, the filter was placed in a 50 -mL tube with $10 \mathrm{~mL}$ of ultrapure water. The tube was vigorously shaken and vortexed before analyzing albuterol concentration in the washings with a spectrophotometer at $276 \mathrm{~nm}$ (BioMate $3 \mathrm{UV}$-visible spectrophotometer, Thermo Fisher Scientific, Waltham, Massachusetts). ${ }^{17}$

\section{Statistical Analysis}

The outcome measure was delivery efficiency, which was calculated as lung dose expressed as percentage of the nominal dose $(2,500 \mu \mathrm{g}$ for all nebulizers). We used analysis of variance followed by Tukey test for multiple comparisons to evaluate differences in delivery efficiency between delivery devices at each site of placement. A paired $t$ test was used to compare delivery efficiency obtained by the same device at 2 different positions and by the same device/position with the NIV operated at 2 different settings. A $P$ value $<.05$ was considered statistically significant. A statistical software package was used for all the calculations (Kaleidagraph 4.1, Synergy Software, Reading, Pennsylvania).

\section{Results}

Data are summarized in Figure 3.

\section{Effect of Device Selection}

Vibrating mesh nebulizers were more efficient than jet nebulizers regardless of their position in the ventilator circuit. Delivery efficiency for the Solo nebulizer when IPAP was $15 \mathrm{~cm} \mathrm{H}_{2} \mathrm{O}$ was $18 \%, 17.6 \%$, and $13.3 \%$ when placed before the mask, before the Y-piece, and at the ventilator, respectively. Delivery efficiency for the Solo nebulizer when IPAP was $20 \mathrm{~cm} \mathrm{H}_{2} \mathrm{O}$ was $14.9 \%, 15.8 \%$, and $10.5 \%$ when placed before the mask, before the Y-piece, and at the ventilator, respectively. Delivery efficiency for the Hudson nebulizer when IPAP was $15 \mathrm{~cm} \mathrm{H}_{2} \mathrm{O}$ was $3.8 \%$ and $3.5 \%$ when placed before the $\mathrm{Y}$-piece and at the ventilator, respectively. Delivery efficiency for the Hudson nebulizer when IPAP was $20 \mathrm{~cm} \mathrm{H}_{2} \mathrm{O}$ was $3.7 \%$ and $2.9 \%$ when placed before the Y-piece and at the ventilator, respectively. The Solo nebulizer was 3.8- and 4.7-fold $\left(\right.$ IPAP $=15 \mathrm{~cm} \mathrm{H}_{2} \mathrm{O}$ ) and 3.6- and 4.0-fold (IPAP $=20 \mathrm{~cm}$ $\mathrm{H}_{2} \mathrm{O}$ ) more efficient than the Hudson nebulizer when placed at the ventilator and the Y-piece, respectively. The NIVO yielded a delivery efficiency of $10.7 \%$ and $9.6 \%$ for IPAPs of 15 and $20 \mathrm{~cm} \mathrm{H}_{2} \mathrm{O}$, respectively. The Solo placed at the mask was more efficient than the NIVO $(P=.032$ and $P=.007$ for IPAPs of 20 and $15 \mathrm{~cm} \mathrm{H}_{2} \mathrm{O}$, respectively), and the Solo placed at the Y-piece was more efficient than the NIVO when IPAP was $15 \mathrm{~cm} \mathrm{H}_{2} \mathrm{O}(P=.004)$. The Solarys performed similarly to the Hudson with both ventilator settings ( $2 \%$ and $2.6 \%$ for IPAPs of 15 and $20 \mathrm{~cm}$ $\mathrm{H}_{2} \mathrm{O}$, respectively).

\section{Effect of Device Position}

The Solo nebulizer placed at the mask or before the Y-piece of the double-limb circuit provided the highest aerosol drug delivery during NIV (14.9-18.4\%). When the IPAP was $15 \mathrm{~cm} \mathrm{H}_{2} \mathrm{O}$, moving the nebulizers from the ventilator to the Y-piece increased delivery efficiency for the Solo $(35 \%, P=.01)$ but not for the Hudson nebulizer. 


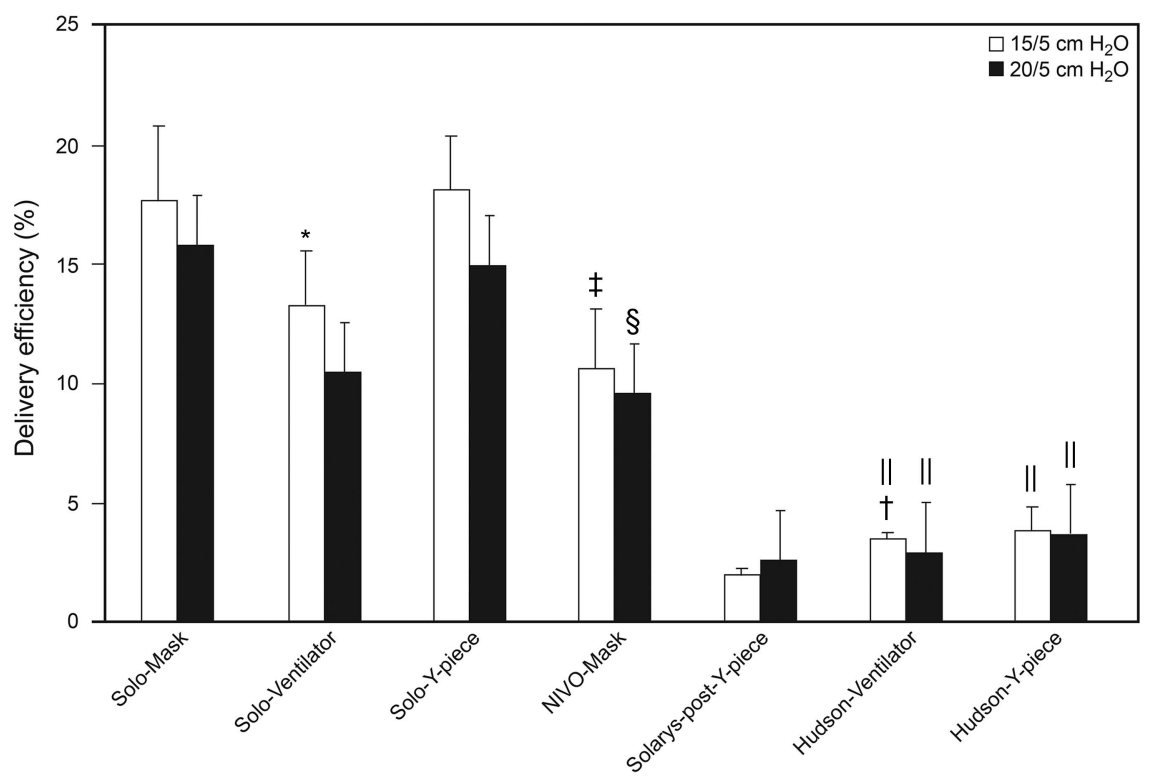

Fig. 3. Delivery efficiency with different devices, ventilator positions, and ventilator settings. Columns represent mean values, and error bars represent standard deviation. ${ }^{*}$ Solo was less efficient at the ventilator than at the Y-piece $(P=.02)$. $†=.01$ when compared to same nebulizer/position with settings $20 / 15 \mathrm{~cm} \mathrm{H}_{2} 0 . \ddagger$ NIVO was less efficient than Solo at the Y-piece $(P=.004)$, and Solo at the mask $(P=.007)$. $\S$ NIVO was less efficient than Solo at the mask $(P=.03)$. \| Hudson was less efficient than Solo regardless of placement and settings $(P<.003)$.

No statistically significant changes were noted when the IPAP was increased to $20 \mathrm{~cm} \mathrm{H}_{2} \mathrm{O}$.

\section{Effect of Increasing IPAP}

Increasing IPAP from 15 to $20 \mathrm{~cm} \mathrm{H}_{2} \mathrm{O}$, keeping the EPAP constant, resulted in a slight decrease in delivery efficiency that was only statistically significant for the Hudson nebulizer placed at the ventilator $(3.5 \%$ vs $2.9 \%$, $P=.01)$ (Fig. 3).

\section{Discussion}

In this study we reported the aerosol delivery efficiency of different aerosol generators placed in different positions in a double-limb circuit of an in vitro anatomically correct model of a child receiving NIV. The vibrating mesh nebulizers were more efficient than jet nebulizers regardless of the position in the circuit. The vibrating mesh nebulizer was more efficient when placed closer to the patient than when placed at the ventilator. Increasing the inspiratory pressure did not improve aerosol delivery efficiency.

This report is the first to investigate aerosol delivery efficiency during NIV using a double-limb circuit; as a result, comparing our results to those of other studies is difficult. Placing the vibrating mesh nebulizer proximal to the patient increased aerosol delivery. In a previous study on aerosol delivery efficiency in a pediatric model of invasive mechanical ventilation, we reported that the vibrating mesh nebulizer placed at the ventilator was the most efficient set-up. ${ }^{5}$ We speculate that the difference may be due to the presence of different degrees of aerosol impaction and size selection. In the case of invasive ventilation, the aerosol generated by the vibrating mesh nebulizer placed before the Y-piece could suffer a greater degree of impaction in the endotracheal tube than the aerosol generated by the nebulizer placed by the ventilator. The latter aerosol suffers more impaction against the circuit walls and the wire as it travels through the circuit, therefore arriving at the endotracheal tube as a smaller aerosol. Several adult studies using a single-limb circuit have shown that a vibrating mesh nebulizer positioned between the exhalation port and the lung model provides efficient drug delivery. ${ }^{7-13}$ These findings are easily explained by the loss of aerosol that occurs through the exhaust port when the aerosol generator is placed proximal to the ventilator. Our findings underscore the importance of not extrapolating findings from one type of ventilation to another. We did not find a difference in output when placing the jet nebulizer from the ventilator before the Y-piece. This is in agreement with data published on invasive mechanical ventilation models. ${ }^{6}$ It was noted that the vibrating mesh nebulizer placed before or after the Y-piece outperformed a vibrating mesh nebulizer that was integrated into the mask. Conversely, an a study using an anatomically correct model with a single-limb circuit showed that delivery of albuterol was most efficient when the vibrating mesh nebulizer was integrated into the mask. ${ }^{5}$ We speculate that the differences could be due to the experimental setup. We used an oral model, whereas they used an oronasal mask. This 
could influence the alignment between the aerosol and either the mouth or the mouth and nose openings.

This study showed that changing the ventilator setting by increasing IPAP did not change albuterol delivery efficiency in a pediatric model of NIV using a double-limb circuit. Conversely, an in vitro adult study using a singlelimb circuit showed an increase in delivery efficiency when IPAP was increased and the nebulizer was positioned after the exhalation port. ${ }^{7}$ We speculate that the difference in findings could be due to the difference in tidal volumes between the pediatric and the adult models.

Our study has limitations that are inherent to the in vitro nature of the study. The drug captured at the filter (lung dose) overestimates the drug reaching the patient because it includes both the inhaled lung dose and part of the exhaled lung dose. However, this is a well-established research methodology. Another limitation of this study is that we tested only one breathing pattern. The findings of this study provide data that may help practitioners choose the right type of aerosol generator and its most efficient placement in a double-limb ventilator circuit during NIV.

\section{Conclusions}

In an in vitro model of a spontaneously breathing child receiving NIV, the effect of nebulizer position on aerosol delivery efficiency depends on the type of device. This study showed that a vibrating mesh nebulizer placed at the mask or before the Y-piece of a double-limb circuit provided the highest aerosol delivery efficiency during NIV. This study also showed that vibrating mesh nebulizers delivered more drug compared to jet nebulizers, regardless of their position in the ventilator circuit. These results underscore the fact that data generated with invasive ventilation models should not be extrapolated to NIV models. In addition, data generated with adult models should not be extrapolated to pediatric models.

\section{REFERENCES}

1. Williams A, Abramo T, Shah M, Miller R, Burney-Jones C, Rooks $\mathrm{S}$, et al. Safety and clinical findings of BiPAP utilization in children $20 \mathrm{~kg}$ or less for asthma exacerbations. Intensive Care Med 2011; $37: 1338-43$
2. Sorofsky A, Klinowski E, Ilgyev E, Mizrachi A, Miller A, Ben Yehuda T, et al. Noninvasive positive pressure ventilation in acute asthmatic attack. Eur Respir Rev 2010;19(115):39-45.

3. Esquinas A, BaHammam A, Scala R, Arie S, Nasilowski J, Siekmeier R, et al. Aerosol therapy during noninvasive mechanical ventilation: review of key technical factors and clinical implications. Can J Resp Ther 2013;49(1):11-18.

4. Scala R. Bi-level home ventilators for noninvasive positive pressure ventilation. Monaldi Arch Chest Dis 2004;61:213-221.

5. White C, Crotwell D, Shen S, Salyer J, Yung D, Zheng J, DiBlasi R. Bronchodilator delivery during simulated pediatric noninvasive ventilation. Respir Care 2013;58(9):1459-1466.

6. Berlinski A, Willis R. Albuterol delivery by 4 different nebulizers placed in 4 different positions in a pediatric ventilator in vitro model. Respir Care 2013;58(7):1124-1133.

7. Chatmongkolchart S, Schettino G, Dillman C, Kacmarek R, Hess D. In vitro evaluation of aerosol bronchodilator delivery during noninvasive positive pressure ventilation: Effect of ventilator settings and nebulizer position. Crit Care Med 2002;30(11):2515-2519.

8. Abdelrahim M, Plant P, Chrystyn H. In-vitro characterisation of the nebulized dose during noninvasive ventilation. J Pharm Pharmacol 2010;62:966-972

9. AlQuaimi M. The effect of using different aerosol devices and masks on aerosol deposition during noninvasive positive pressure ventilation in an adult lung model. Respiratory Therapy Thesis. 2011. Paper 5. Georgia State University (Atlanta, Georgia). http:// scholarworks.gsu.edu/rt_theses/5.

10. Calvert L, Jackson J, White J, Barry P, Kinnear W, O'Callaghan C. Enhanced delivery of nebulized salbutamol during noninvasive ventilation. J Pharm Pharmacol 2006;58:1553-1557.

11. Dai D, Kang J, Sun L, Tan W, Zhao H. Influence of exhalation valve and nebulizer position on albuterol delivery during noninvasive positive pressure ventilation. J Aerosol Med Pulm Drug Deliv 2013;26: $1-8$.

12. Michotte J, Jossen E, Roeseler J, Liistro G, Reychler G. In vitro comparison of five nebulizers during noninvasive ventilation: analysis of inhaled and lost doses. J Aerosol Med Pulm Drug Deliv 2014;27:430-440

13. Parkes S, Bersten A. Aerosol kinetics and bronchodilator efficacy during continuous positive airway pressure delivered by face mask. Thorax 1997;52:171-175.

14. Dolovich MB, Dhand R. Aerosol drug delivery: developments in device design and clinical use. Lancet 2011;377(9770):1032-1045.

15. Mitchel J, Avvakoumova V, Schneider H, Ali R, Nagel M. The evaluation of a new continuous liquid aerosol delivery system for the patient on mechanical ventilation. Respir Drug Delivery 2013; 373-378.

16. Bickmann D, Wachtel H, Kröger R, Langguth P. Examining inhaler performance using a child's throat model. Respir Drug Delivery 2008;2:565-570.

17. Chavez A, McCraken A, Berlinski A. Effect of face mask static dead volume, respiratory rate and tidal volume on inhaled albuterol delivery. Pediatr Pulmonol 2010;45(3):224-229.

This article is approved for Continuing Respiratory Care Education credit. For information and to obtain your CRCE

(free to AARC members) visit

www.rcjournal.com

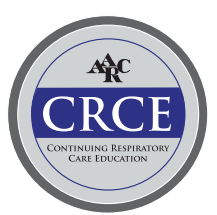

